\title{
Effective Relay-Node Selection for Clustering Ad Hoc Network
}

\author{
Kang-Whan Lee *, Yun Chen * and Jang-Sup Shim ** \\ *Department of Computer Engineering, Korea University of Technology and \\ Education, Cheonan, Republic of Korea \\ ** Corresponding Author, Institute for Information \& communications Technology \\ Promotion (IITP), Daejeon, Republic of Korea/sjs@iitp.kr \\ kwlee@koreatech.ac.kr
}

\begin{abstract}
In ad hoc network, the scarce energy management of the mobile devices ha ve become a critical issue in order to extend the network lifetime, therefore, the energy consumption is important in the routing design, otherwise cluster schemes are efficient in energy conserving. In this paper, we consider a novel routing protocol using the cluster schemes to find the optimal energy routing path based on a special topology structure RODMRP, RODMRP is a hierarchical ad hoc network structure which combines the advantage of the tree based and the mesh based network. This scheme divides the nodes in different level found on the node energy condition, and the clustering is established based on the levels. This protocol considered the residual energy of the nodes and the total consuming energy ratio on the routing path to get the energy efficiently routing.
\end{abstract}

Keywords: Mobile ad hoc network, Energy, Cluster, Adaptive, Transmit power

\section{Introduction}

An ad hoc network is a mobile self-organized network with dynamic topology. In addition, every node can serve as a router. In ad hoc network, especially in a sensor network, the wireless device power is provided by a battery and the network node energy is limited. Therefore it becomes very important to use the node energy in high efficiency for prolonging the network lifetime as long as possible [1].

For the aim of improving the networks lifetime and capability, we should reduce the node energy expending and routing link cost. That is why establishing the network structure and choosing the routing path are very important in the ad hoc network. Above the reasons, in this paper an Energy-conserving Context-awareness Clustering-algorithm (ECC) has proposed to establish the network clustering structure, and a routing algorithm is Introduced to choose the Optimal Energy Routing Protocol (OERP) path. Because in ad hoc network, the topology, nodes residual energy and energy consuming rate are dynamically changing. The network system should react continuously and rapidly to the changing conditions, and make corresponding action according to different conditions. So we use the context aware computing to actualize the cluster head node, the routing path choosing. In this paper, we show a new Energy-conserving Context-awareness Clustering-algorithm (ECC). This algorithm discusses several energy factors: transmit power, node mobility, and node residual energy [2][3][4]. Totally thinking of the factors, we chose the nodes which have low transmit power to cover at least $\mathrm{N}$ neighbors, more residual energy and low mobility as the cluster head node. To save the energy, before the clustering performing, we use a transmitting adaptive scheme.

This paper is organized as follows: In section II, we show the rule based context aware architecture for MANET, in section III, we present the Energy-conserving Context-awareness Clustering-algorithm (ECC), the Optimal Energy Routing 
Protocol algorithm (OEPR), in section IV, we present the Optimal Energy Routing Protocol algorithm (OEPR), and the last part is the conclusions.

\section{Context-Awareness Architecture for Energy Conserving Routing In Manet}

In this paper, a rule based context-awareness architecture is designed to perform network clustering foundation and networking routing actualizing. Also, this paper proposes the context-awareness architecture using the ECA (Event-ConditionAction) rules and designs the main architecture while considering the list below.

Several problems are considered in this paper :

- Establishing the cluster.

- Maintenance of the cluster structure.

- Choosing the routing path in improving the energy consumption

- Information elements included to design the context rules.

These problems will be resolved at the next section

The algorithm we proposed in this paper is performed in a particular network structure RODMRP Structure which is the Resilient Ontology-based Dynamic Multicast Routing Topology for ad hoc network[5][6]. This topology combined the tree-based structure and mesh-based structure that take both advantage of tree-based structure and mesh-based structure, hence this structure is resilient topology than the others' structure. Therefore, in this paper, we apply the proposed protocol in RODMARP model [3].

\section{ECC Algorithm for Energy Conserving}

This section introduces the clustering maintenance including the cluster heads maintenance and cluster member maintenance. The clustering algorithm of Energy Conserving Clustering (ECC) model divides the geographical region to be covered into small zones. The essential operation in mobile node for clustering is to select a set of cluster heads among the nodes in the network, and the cluster heads are responsible for coordination with their clusters (intra-cluster), and communication with other cluster heads (inter-cluster).

Clustering is a known technique for grouping nodes that are close to one another in a network and it has the unique potential to reduce useful energy consumption. So in this paper, an energy context clustering approach is used. Otherwise, in this paper, the cluster technique use weight-based for multi-hops ad hoc network, which nodes use weight cost function based on residual energy and node mobility and the number of neighbor nodes to form clusters and choose a cluster-head[7][8][9][10][11].

\subsection{Clustering Model for Energy Conserving}

These algorithms consider the following aspects: choosing the node which has more residual energy, low mobility, and low transmit power to be the cluster head.

In this below shows, the ECC algorithm flow chart the relay node selection technique utilizing an energy conserving operation. 


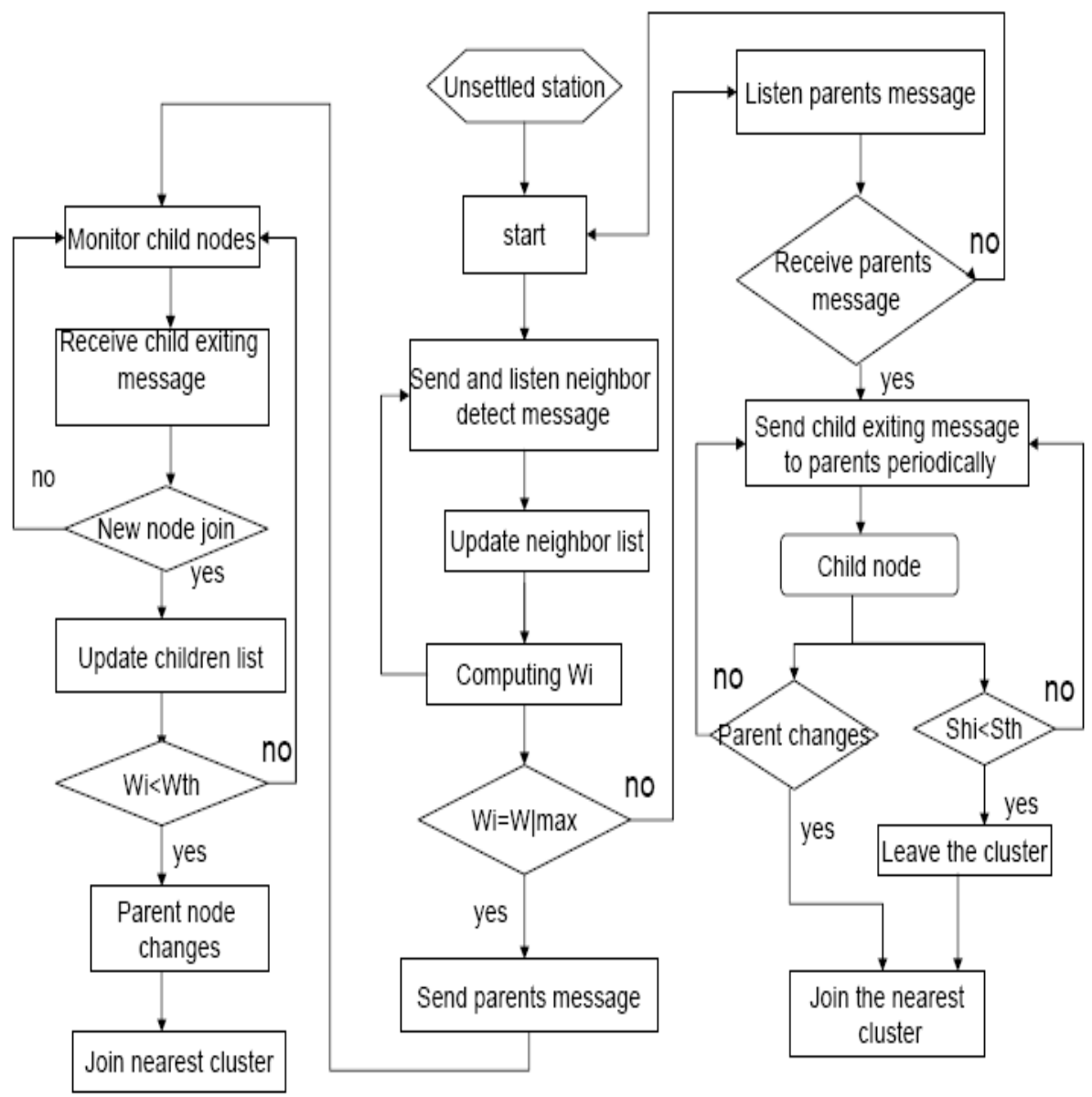

Figure 1. ECC Algorithm Flow Chart ECC

The efficient use of the scarce energy resources available to ad hoc and sensor network nodes is one of the fundamental tasks of the network designer. Since nodes consume a considerable amount of energy to transmit/receive messages, reducing the energy consumed for radio communications is an important issue. Refer to specific wireless channel and energy consumption models. For simplicity, assume the radio signal propagates according to the free space model and that we are interested in minimizing the transmit power only. With these assumptions, the power needed to send the message directly from $u$ to $v$ is proportional to $d^{2}$; in case the packet is relayed by node $w$, the total power consumption is proportional to the node distance each other's.

Table. 1 defines the notations applied in the algorithm. The velocity is based on the signal strength between the node $i$ and its neighbors. And the following example assumes that each node gets the 1-hop and 2-hop nodes as neighbor nodes. This section introduces ECC (context aware weighting clustering algorithm).

Table 1. Notations Define in ECC

\begin{tabular}{|c|c|}
\hline Notation & Descriptions \\
\hline$i$ & Node ID \\
\hline$E_{i}$ & Residual energy of node $i$ \\
\hline$P_{i}$ & Transmit power of node $i$ \\
\hline$d_{i j}$ & Distance between node $i$ and node $j$. \\
\hline
\end{tabular}




\begin{tabular}{|c|c|}
\hline$V_{i}$ & Velocity of node $i$ \\
\hline$N_{i}$ & Number of neighbors of node $i$ \\
\hline$S_{i j}$ & Signal strength from node $i$ to node $j$ \\
\hline$S_{t h}$ & Predefine threshold value of $S_{i j}$ \\
\hline$W_{i}$ & ECC Weighting value of node $i$ \\
\hline
\end{tabular}

\subsection{Clustering Model Algorithm description}

Each node in the network contains a neighbor nodes list, and updates the neighbor nodes list based on the interaction detective message between each node.

The ECC weighting value $W i$ is defined as (1)

$$
\mathbf{W i}=\mathbf{E} \mathbf{i}^{\mathrm{x} 1} * \mathbf{P i}^{-\mathrm{x} 2} * \mathbf{V i}^{-\mathrm{x} 3}
$$

\section{Maintenance of Parents nodes}

If residual energy of parents nodes $W_{i}<W_{t h}, W_{t h}$ can be defined as $\beta$ average $\left.W_{i}\right\}$, $(0<\beta<1)$. Parent nodes send parent nodes changing message to its children, the cluster head changes, and join the nearest cluster.

If cluster parent node can't receive exiting message from its children nodes, parent node updates children nodes list, delete the children nodes information.

\section{Maintenance of Children nodes}

If the children nodes receive parent nodes change message, then children nodes leave this cluster, to join the nearest cluster. The children nodes detect the signal strength less than predefine threshold value, the children nodes will not send exiting message to parent nodes, then leave this cluster and join the new nearest cluster.

Table 2. Weighting Value Factors

\begin{tabular}{|l|l|l|l|}
\hline Condition & $\boldsymbol{X} \mathbf{X}$ & $\boldsymbol{X} \mathbf{2}$ & $\boldsymbol{X 3}$ \\
\hline Average $V i$ is very low & 1 & 1 & 0 \\
\hline Average $V_{i}$ is high & 1 & 1 & 0.5 \\
\hline Average $E_{i}$ is high & 0 & 1 & 1 \\
\hline
\end{tabular}

The notation of $X 1, X 2, X 3$ are the weighting value factors, in general network, the three energy elements (Residual energy of node, transmit power, velocity of nodes) is equally important to the energy consumption. In different conditions, the $X 1, X 2, X 3$ can be defined different values which is shown in Table.2.

\subsection{Clustering ECC Algorithm Rules}

Each node have:

ECC algorithm rules are given as follows:

Step1. Each node $i$ sends neighbor detective message, and listens to other nodes

Step2. Computing $E_{i}, P_{i}$ and $V_{i}$, get the ECC value $W_{i}$.

Setp3. Computing the $W_{i}$, if the $W_{i}=\left.\mathrm{W}\right|_{\max }$. 


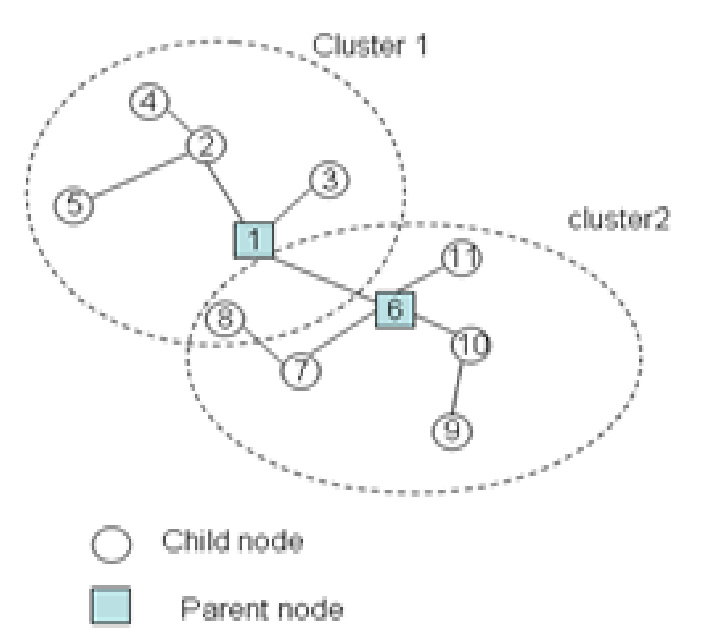

Figure 2. ECC Cluster Structure from RODMARP

This paper proposes the context aware architecture to classify the context data into different elements, and an illustration of ECC using classification data is shown as Figure 2 and Table.3. In this simple illustration, the context classification covers six elements: residual energy, transmit power, velocity, number of neighbors, ECC weighting value, and the condition (shown in Table.3). In this illustration, we predefine $X 1=X 2=X 3=1$. According equation (1), get the $\mathrm{ECC}$ value, decide the node condition \{cluster head: cluster member\} based on the ECC weighting value $W i$. The network cluster structure is shown as Figure 4, in cluster 1, node 1 as the parent node, and node 2,3,4,5 children nodes, and node 6 as neighbor node.

Table 3. Context Classification Form

\begin{tabular}{|c|c|c|c|c|c|c|}
\hline$I d$ & $E i$ & & $P i$ & $V i$ & $W i$ & Condition \\
\hline 1 & 5 & & 10 & 10 & 0.05 & parent node \\
\hline 2 & 4 & & 20 & 20 & 0.01 & Child node \\
\hline 3 & 6 & & 10 & 20 & 0.02 & Child node \\
\hline 4 & 4 & & 20 & 10 & 0.02 & Child node \\
\hline 5 & 3 & & 20 & 20 & 0.075 & Child node \\
\hline 6 & 6 & & 10 & 15 & 0.4 & Neighbor node \\
\hline
\end{tabular}

\section{Simulation Results}

For the total path, we considered the link cost as a weighting value $\mathrm{C}$. At the current moment, the lifetime of node $\mathrm{i}$ can be define as $T_{i}=\operatorname{Min}\left(\frac{\mathrm{E}_{\mathrm{i}}}{\sum \underset{\mathrm{j} \in \mathrm{N}}{\mathrm{d}_{\mathrm{j}}^{\alpha}}}\right), T_{\operatorname{Min}}=\operatorname{Min}\left(\mathrm{T}_{\mathrm{i}}\right)$ is the node which has shortest lifetime on the path $\mathrm{p}, \mathrm{P}$ is the set of routing paths from the source to destination. In the weighting function, one of the parameters is $\mathrm{T}_{\min }$, another parameter is the ratio value of the sum of path loss and the sum of residual energy.

The link cost is shown as (2)

$$
C=\left[\operatorname{Min}\left(\frac{E_{i}}{\sum d_{i}^{\alpha}}\right)\right]^{-Y_{1}}+\left[\frac{\sum d^{\alpha}}{\sum E_{i}}\right]^{Y_{2}}
$$


If the average residual energy is very high, the minimum path loss algorithm can be used, if the average residual energy is very low, the OERP algorithm optimal energy routing protocol algorithm (OEPR) can be used.

\section{Performance Evaluation}

In order to estimate the efficiency of the proposed protocol for mobile ad hoc network, we use Ns2, which run in circumstance Windows+Cygwin+ns2 to make the simulation.

The simulation parameters are listed as follows in Table 4.

Table 4. Simulation Parameters

\begin{tabular}{|l|l|}
\hline \multicolumn{2}{|c|}{ Simulation parameters } \\
\hline Number of nodes & $20 \sim 80$ \\
\hline Physical area & $670 * 670$ \\
\hline velocity & $0 \sim 5 \mathrm{~km} / \mathrm{h}$ \\
\hline MAC & 802.11 \\
\hline Experiment time & 900 \\
\hline
\end{tabular}

In the simulation we make a comparison between the AODV and our proposed OERP. And consider three network evaluation parameters: network lifetime (average lifetime), delay time and the delivery ratio which follow the changes of traffic load and the node density. The comparison is shown as the following figures.

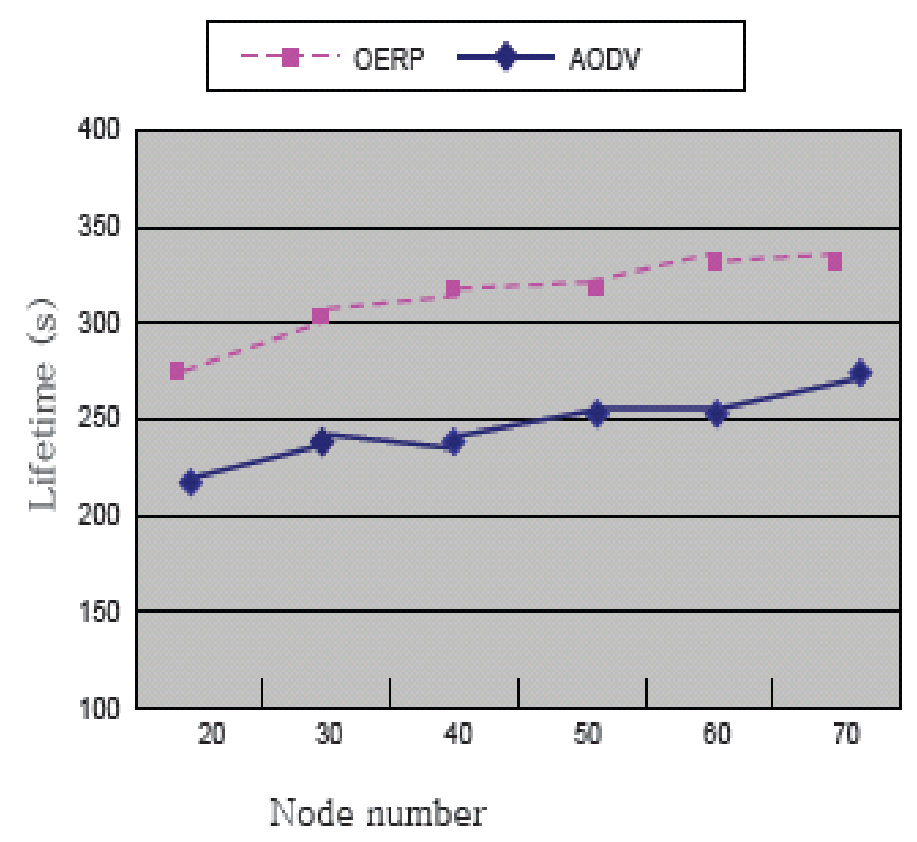

Figure 3. Evaluation of Lifetime 


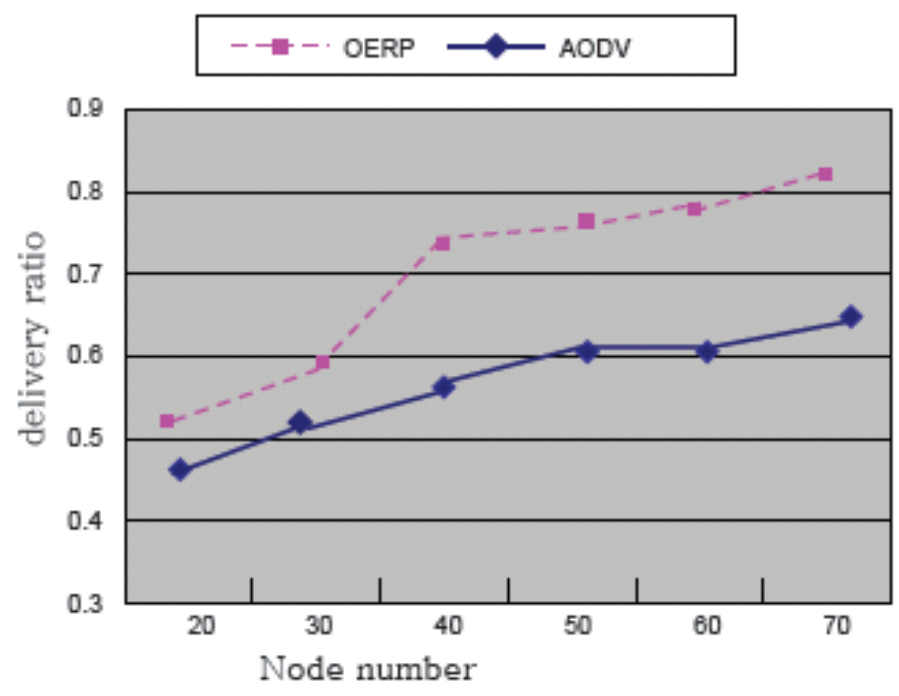

Figure 4. Evaluation of Delivery Ratio

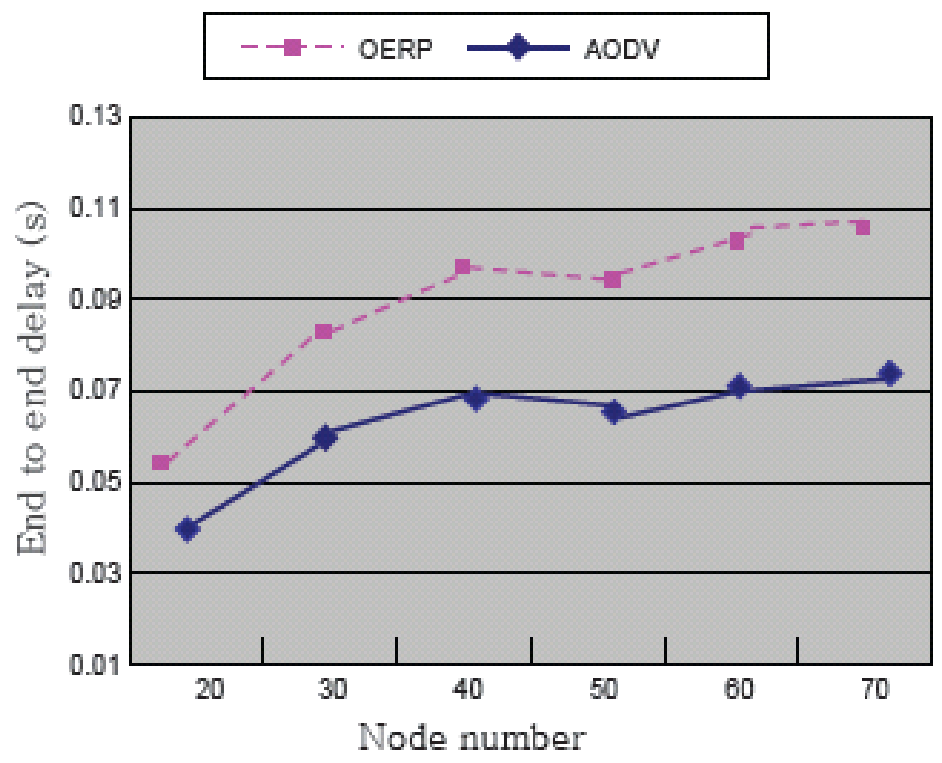

Figure 5. Evaluation of Delay Time

\section{Conclusions}

This paper proposed Energy-conserving Context-awareness Clustering-algorithm (ECC) and an Optimal Energy Routing Protocol (OERP) for the ad hoc network. The proposed ECC, OERP algorithms synthetically considered the energy elements to improve the energy conserving in ad hoc network. The proposed ECC and OERP could need to know the network topology and residual energy of every node. It would be difficult to perform in large size networks. Otherwise, every node should broadcast its residual energy to other nodes. These parameters of energy consumption would also be considered and analyzed as the future works. 


\section{Acknowledgments}

This research was supported by the MSIP (Ministry of Science, ICT and Future Planning(2014H1C1A1066391), Korea, under the Specialized Co-operation between industry and partially supported by the Education and Research Promotion Program of KUT.

\section{References}

[1] R. Agarwal and M. Motwani, "Survey of cluster of clustering algorithms", International journal on Computer Science and Engineering, vol. 1, no. 2, (2009).

[2] Y. Chen and K. Lee, "A study on the Context-Awareness architecture for Energy Conserving Routing in MANET", Journal of information and communication convergence engineering, vol. 1, (2007).

[3] S. G. Kim and K. W. Lee, "A study on the advanced inference routing network scheme for RODMRP", International Conference on Advanced language Processing and Web Information Technology, (2008).

[4] Y. J. Oh and K. W. Lee, "Energy conserving routing algorithm based on the direction for Mobile Adhoc network", Proceedings Conference on Information and Communication Engineering, vol. 17, no. 1, (2013).

[5] J. J. Lee and J. S. Lim, "Effective and Efficient Jamming Based on Routing in Wireless Ad Hoc Networks", IEEE Communication Letters, vol. 16, no. 11, (2012).

[6] Y. J. Oh, D. K. Oh and K. W. Lee, "A Study Optimal Path Availability Clustering Algorithm in Ad Hoc Network", Future Information Communication Technology and Applications Lecture Notes in Electrical Engineering, vol. 235, (2013).

[7] Y. J. Oh, D. K. Oh and K. W. Lee, "A Clustering Algorithm Based on Mobility Properties in Mobile Ad Hoc Network", International Journal of Distributed Sensor Networks, vol. 2015, (2015).

[8] S. Soro and W. B. Heinzelman, "Cluster head election techniques for coverage preservation in wireless sensor networks", Ad Hoc Networks, vol. 7, issue 5, (2009).

[9] A. S. M. S. Hosen, S.-H. Kim, H.-H. Cho, "An Energy Efficient Cluster Formation and Maintenance Scheme for Wireless Sensor Networks", Journal of information and communication convergence engineering, vol. 10, no. 3, (2012).

[10] ] T.-H. Kim, D. Tipper and P. Krishnamurthy, "Localized Algorithm to Improve Connectivity and Topological Resilience of Multi-hop Wireless Networks", Journal of information and communication convergence engineering, vol. 11, no. 2, (2013).

[11] X. Yang and Y. C. Kiat, "Measuring Group Mobility: A Topology Based Approach, Wireless Communications Letters", IEEE, vol. 2, Issue 1, (2013).

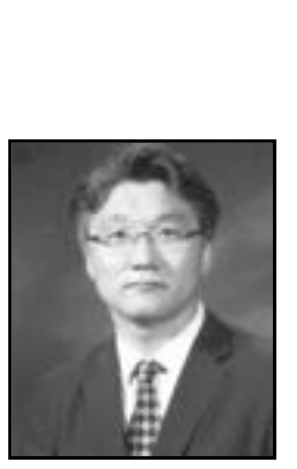

\section{Authors}

Kang-whan Lee, he received his B.S. and M.S. Degrees in Electronics Engineering from Hangyang University and Chung-Ang University, Korea, in 1987 and 1989, respectively. He earned his PhD in Electronics Engineering from Chung-Ang University, Korea. And he has finished course work from Korea Advance Institute Technology(KAIST) in 1996. Also, he has worked as an invited professor on CICA (Center of Institute Communication Association), France in 1997. Since 1989 he has been a member of Senior Technical Staff at Video Communications section of Electronics and Telecommunications Research Institute(ETRI). He is currently a professor at Korea University of Technology and Education. His main research is Wireless Sensor Network, Ad-hoc network, and Mobile Telecommunications-Advanced UoC (Ubiquitous System on Chip) with Wireless SoC. 


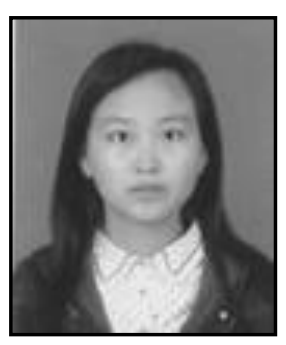

Yun Chen, she received her B.E. Degree in Electronics Information Science and Technology from Shandong University, China, in 2006. She is currently taking her M.S course at the School of Information Technology of Korea University of Technology and Education. Her master major is Computer Engineering, and now she is studying the energy conserving in MANET and context aware system for wireless UoC system design. Her email address is fei822@kut.ac.kr.

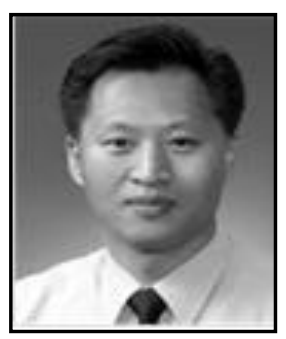

Jang-Sup Shim, he received his $\mathrm{PhD}$ Degree in Computer Sciences Engineering from Chung Buk National University, Korea in 2005. He is currently working as the Research Fellow on IITP. He has over 30 years of professional experience in areas related to digital communication, intelligent network, database handling technology and policy making related to the information and telecommunication sector. $\mathrm{He}$ is also experience in areas related to research and development management by public area. He is the author of several research papers in the area of information and communication processing among the database. He has been active in academia activities that is KIICE (The Korea Institute of the Information and Communication Engineering), KIPS(Korea Information Processing Society) and Member of Institute of Electrical and Electronics Engineers(IEEE). 
International Journal of Control and Automation

Vol. 10, No. 1 (2017) 